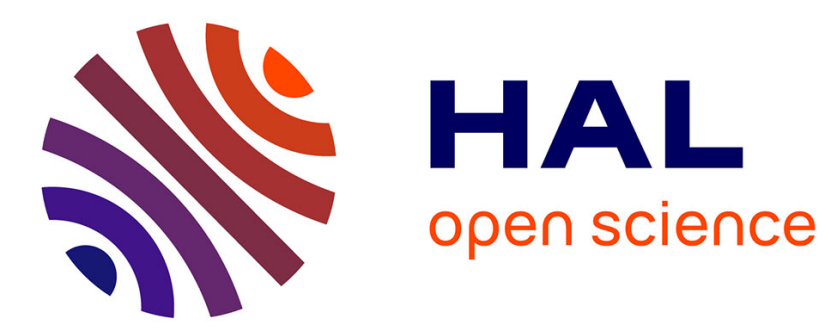

\title{
CONFINED PROPAGATION OF NEUTRONS IN SOLID WAVEGUIDES (FIBRES)
}

\author{
R. Alvarez-Estrada, M. Calvo
}

\section{To cite this version:}

R. Alvarez-Estrada, M. Calvo. CONFINED PROPAGATION OF NEUTRONS IN SOLID WAVEGUIDES (FIBRES). Journal de Physique Colloques, 1984, 45 (C3), pp.C3-243-C3-248. 10.1051/jphyscol:1984341 . jpa-00224055

\section{HAL Id: jpa-00224055 https://hal.science/jpa-00224055}

Submitted on 1 Jan 1984

HAL is a multi-disciplinary open access archive for the deposit and dissemination of scientific research documents, whether they are published or not. The documents may come from teaching and research institutions in France or abroad, or from public or private research centers.
L'archive ouverte pluridisciplinaire HAL, est destinée au dépôt et à la diffusion de documents scientifiques de niveau recherche, publiés ou non, émanant des établissements d'enseignement et de recherche français ou étrangers, des laboratoires publics ou privés. 


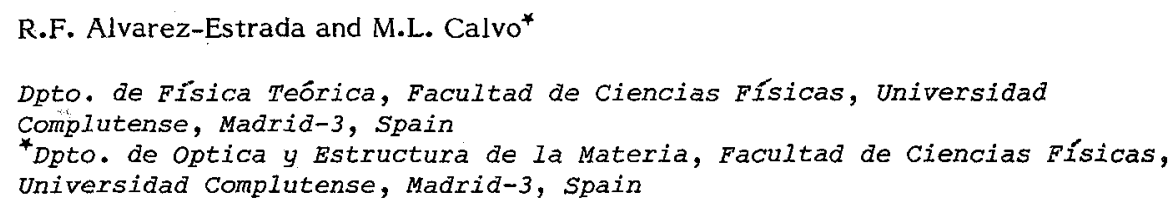

\section{RESUME}

L'iđáe de fibres à neutrons, proposée dans un article récent, est passée brièvement en revrue. De nouveaux résultats sont présentés, en particulier sur: (a) les fibres aimantées, (b) les modes de propagation dans des fibres circulaires homogènes (limites supérieures sur leur nombre, approximations quasi-classiques), (c) sur 1'absorption des neutrons par les noyaux.

\section{ABSTRACT}

The physical possibility of neutron fibres, proposed in a previous paper, is reviewed briefly. Further possibilities and results are reported specifically about: (a) magnetized fibres, (b) propagation modes in homogeneous circular fibres (upper bounds on their number, quasiclassical approxi mations), (c) neutron absorption by nuclei.

\section{SUMMARY OF PREVIOUS WORK}

In [1], we have conjectured the possible confined propagation of themal neutrons along cylindrical solid (non-hollow) fibres of small cross section, made up by certain elements $(T i, M n, \ldots)$ such that the overall amplitude (b) for the low-energy muclear scattering of neutrons on them be negative. In [1], among other topics, we studied specifically:

(i) Geometrical-optics descriptions and procedures for confin ing neutrons,

(ii) the possibility that Ti fibres (whose length be about 5 to $10 \mathrm{~cm}$.) could have applications for slow neutron radiotherapy,

(iii) the (rather theoretical) possibility that fibres made up by magnetically saturated co could propagate fully polarized neutrons, even if their length would be rather limited by the relatively large nei 
tron absorption by Co. The neutron wavefunction $\psi(\bar{x})$ for a confined propa tion mode with energy $E$ in a solid fibre along the z-axis fulfills the Schroedinger equation (meneutron mass, $\not h=P l$ lanck's constant, $\bar{x}=(\underline{x}, z), r=$ $=|\underline{x}|)$ :

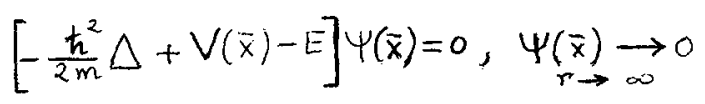

with $v(\bar{x})=0$ outside the fibre. For a homogeneous fibre having $p$ nuclei per unit volume, the potential "seen" by the neutron inside the fibre is [2] $V(\bar{x})=2 \pi h^{2} \rho \mathrm{bm}^{-1}, \mathrm{~b}(\mathrm{Re} \mathrm{b}<0)$ being the neutron-nucleus scattering ampli tude. When lattice periodicity is taken into account, $V(\bar{x})$ inside the fibre is to be replaced by the standard sum of Fermi pseudopotentials [3]. In [1], we also studied:

(iv) the neutron propagation modes $\psi$ for homogeneous fibres with circular cross section, without or with nuclear absorption,

(v) the effects of lattice periodicity, without nuclear absorption, upon the propagation modes, namely, wide allowed and small forbidden bands for the neutron along the fibre.

\section{MAGNETIZED FIBRES FOR CONFTIED PROPAGATION OF NEUTRONS}

Magnetized fibres should satisfy some reasonable conditions:

(i) They should be ferromagnetic or, at least, ferrimagnetic , so that in the ordered state they have a non-vanishing net magnetization, which should lie along the fibre axis: if possible, their curie tempera ture $T$ should lie well above ordinary temperature (so that their eventual use be not restricted to low temperatures) and they should be magnetically saturated. ii) If possible, the (real part of the) overall nuclear scattering amplitude, $b$, should be negative, and, anyway, if $b_{\text {mag }}$ denotes the net magnetic scattering amplitude (see chapter 6 in [4]), then at least (the real part of) one of the two amplitudes $b \pm b_{\text {mag }}$ has to be negative.

(iii) The overall linear absorption coefficient $\mu$ for neutrons (see Table 6 in [4]) should not be large, in order to avoid an excessive nuclear absorption. If only one of the amplitudes $b+b_{\text {mag }}$ is negative, then the confined neltrons propagating along the fibre would be fully polarized, wille if both are negative, neutrons with different polarizations would propagate with different propagation constants (birefringence). Here, we shall concentrate on fibres containing Mn. One interesting possibility ful filling all the above requirements would be a fibre made up by the ferrimagnetic compound $\mathrm{Mn}_{4} \mathrm{~N}$. In fact:

(i) The unit cell in a magnetized sample of $\mathrm{Mn}_{4} \mathrm{~N}$ has a net magnetic moment of 1.2 Bohr magnetons [5] and $\mathrm{T}_{\mathrm{C}}\left(\mathrm{Mn}_{4} \mathrm{~N}\right)=743^{\circ} \mathrm{K}[6]$. 
(ii) By using Table 2 in [4], one finds $4 b(\mathrm{Mn})+b(\mathrm{~N})=-0.5 \times 10^{-12}$ cm., so that confined neutrons with both spin projections could propagate in a completely urmagnetized $\mathrm{Nn}_{4} \mathrm{~N}$ fibre: moreover, the magnetic scattering amplitude per unit cell corresponding to a net magnetic mament of 1.2 Bohr magnetons equals $1.1 \times 10^{-12} \mathrm{~cm}$. and, hence, it exceeds the net (negative) nuclear amplitude per unit cell, so that confined neutrons could propagate in a $\mathrm{Mn}_{4} \mathrm{~N}$ fibre magnetized along the z-axis only when their spins are anti parallel to the net magnetization.

(iii) Since $\mu(\mathbb{N}) \simeq 0$ (Table 6 in $[4]$ ), the overall linear absorption coefficient for $\mathrm{Mn}_{4} \mathrm{~N}$ is determined by that of $\mathrm{Mn}\left(\mu(\mathrm{Mn}) \simeq 0.6 \mathrm{~cm}^{-1}\right)$, which is not large. Fibres made up by the compound $\mathrm{Mn}_{2} \mathrm{Sb}$ would provide another possibility: $2 \mathrm{~b}(\mathrm{Mn})+\mathrm{b}(\mathrm{Sb})=-0.18 \times 10^{-12} \mathrm{~cm}$., so that the net nuclear amplitude is negative. The ferrimagnetic structure of $\mathrm{Mn}_{2} \mathrm{Sb}$ [7] leads to a net magnetic moment of 1.74 Bohr magnetons per unit cell: then, the net magnetic scattering amplitude per unit cell is $1.6 \times 10^{-12} \mathrm{~cm}$. As $\mu(\mathrm{sb}) \simeq 0.1 \mathrm{~cm}^{-1}$, the overall linear absorption coefficient of $\mathrm{Mn}_{2} \mathrm{Sb}$ is not large.

Finally, fibres made up by materials which become antiferromag netic below the Neel temperature (with vanishing net magnetization) could also allow for confined propagation of neutrons, provided that their over all nuclear amplitudes be negative and their overall linear absorption coefficients be not large. Some examples are: (i) Mn $S$, where $b(\mathrm{Mn})+\mathrm{b}(\mathrm{s})=$ $=-0.05 \times 10^{-12} \mathrm{~cm}$., a rather small value, and $\mu(\mathrm{s})=0.011$, (ii) $\alpha-$ manganese.

\section{PROPAGATION MODES IN HOMOGENEOUS CIRCUIAAR FIBRES: FURTHER RESULTS}

For a hamogeneous circular solid fibre of radius $\mathrm{R}$ without nu clear absorption ( Im $b=0)$, in cylindrical polar coordinates $(r, \phi, z)$, the propagation modes read $\psi(\bar{x})=g_{n M}(r) \exp i(\beta z+M \phi), M=0, \pm 1, \pm 2, \ldots$, $n=1,2, \ldots, \beta$ being the real propagation constant. The number of bound sta tes of a quantummechanical spinless particle of mass $m$ in a two-dimensio nal azimuthally-symmetric potential $V(r)$ with $M \neq 0$ is less or equal than $(2|\mathrm{M}|)^{-1} 2 \mathrm{~m} \not^{-2} \int_{0}^{\infty} \mathrm{dr} . r|\mathrm{~V}(\mathrm{r})|$. This upper bound can be proven by extending directly to the two-dimensional case the proof of Bargmann's three-dimensional bound (see, for instance, [3]). For the actual $v(r)$, and by including a factor 2 to count both neutron spin projections, the above bound yields the following bound on the number of neutron propagation modes $N(M)$ with $M \neq 0: N(M) \leq|M|^{-1} \cdot 2 \pi R^{2} \rho(-b)$. That is, there may be neutron propagation modes if $|M| \leq 2 \pi R^{2} \rho(-b) \equiv M_{\max }$ and there is none, certainly, if $|M|>M_{\max }$. For typical values $\rho \simeq 10^{22}$ nuclei/ $\mathrm{cm}^{3},-b \approx 10^{-12}$ cm. , $2 \pi R^{2} \approx 10^{-8}$ to $10^{-7} \mathrm{~cm}^{2}$., one finds $M_{\max } \approx 10^{2}$ to $10^{3}$. 
By applying the quasiclassical quantization conditions for the bound state energies in two-dimensional potentials [9], one gets the dispersion relation between $E$ and $\beta$ for the neutron propagation mo des with given $M \neq 0\left(2 m h^{-2} E-\beta^{2}=-x^{2}, q(x)=\left[4 \pi \rho(-b)-x^{2}\right]^{1 / 2}, n=1,2, \ldots\right)$ :

$$
\left[q(x)^{2} R^{2}-M^{2}\right]^{1 / 2}-M[\pi / 2-\arcsin (M / q(x) R)]=(n-1 / 2) \pi, \quad|M|<q(x) R
$$

which can be expected to hold for $\mathrm{R} \geq 10^{-4} \mathrm{~cm}$.

In turn, the number of propagation modes with given $M \neq 0$ can be obtained, in quasiclassical approximation, from (2):

$$
N(M) \simeq \frac{2}{2 \pi}\left\{\left[q(0)^{2} R^{2}-M^{2}\right]^{1 / 2}-M[\pi / 2-\arcsin (M / q(0) R)]\right\}
$$

with $|M|<R . q(0)$. A factor 2 has been included to count neutrons with both spin projections.

\section{CONF'TNED PROPAGATION AND NUCLEAR ABSORPTION IN FIBRES: FURTHER REMARKS}

- Let us recall that in a hollow neutron guide having rectangular cross section of inner dimensions $a, b$ the neutrons penetrate a distance $\mathrm{d}=100 \AA$ into the inner walls $[10]$. Then, a very rough way of estimating the $r$ adiative neutron capture by nuclei in solid fibres having, say, cir cular cross section of radius $R$, consists in imposing that

$\rho(s f) \sigma_{r c}(s f) \pi R^{2}<2(a+b) d \sigma_{r c}(h g) \rho(h g)$, where $\sigma_{r c}(h g)\left(\sigma_{r c}(s f)\right)$ is the (suit ably averaged) cross section for radiative capture of themal neutrons by the nuclei making up the inner walls of the hollow guide (the solid fibre). $\rho(\mathrm{hg}), \rho$ (sf) are the average numbers of nuclei per unit volume for the hollow guide wall and/ fibre, respectively. For typical values $a+b \approx 20$ to $25 \mathrm{~cm}$. and $\left[\rho(h g) \sigma_{r c}(h g)\right] /\left[\sigma_{r c}(\mathrm{sf}) \circ(\mathrm{sf})\right] \approx 10^{-1}$ to 1 , we see that the fibre radius is $\mathrm{R} \approx 10^{-3}$ to $4 \times 10^{-3} \mathrm{~cm}$. A somewhat smaller value of $\mathrm{R}$ could, then, reduce the radiative neutron capture and, hence, the resulting $r$-ray flux, in solid fibres.

- We shall consider briefly the nuclear absorption effects on the confined propagation modes for neutrons in semiinfinite fibres made up by perfectly crystalized lattices. The latter have cross sections $\Omega$ in the $\underline{x-p l a n e}$ and extend fram $z=0$ up to $z=+\infty$. Now, $v(\bar{x})=V(x, z)$ is the stan dard sum of all Fermi pseudopotentials centered at all lattice points inside the fibre, so that $V(\underline{x}, z)=V\left(\underset{x}{x}, z+1\left|\bar{a}_{3}\right|\right), z>0,1=1,2,3, \ldots,\left|\bar{a}_{3}\right|=a$ being the fundamental translation vector along the fibre $(z-)$ axis. Since nuclear absorption is allowed, $b$ is complex, with $\operatorname{Re} b<0, \operatorname{Im} b<0$. In this case, the neutron propagation modes $\psi$ satisfy $\mathrm{Eq}$. (1) and bear the form $\psi(\bar{x})=\exp i \beta z \cdot U(\underline{x}, z)$, where the quasimomentum $\beta$ is complex (Im $\beta$ charac 
terizing the attenuation along the fibre) and $U(\underline{x}, z)$ vanishes for $r=|\underline{x}| \rightarrow \infty$ and is periodic in $z$ with period $\left|\bar{a}_{3}\right| \cdot A$ direct generalization of the devel opments in [1], section 4, yields the exact representation:

$\operatorname{Im} \beta=\left[\frac{m}{\hbar^{2}} \int_{\Omega} d^{2} \underline{x} \int_{0}^{a} d z \operatorname{Im} v(\underline{x}, z)|U(\underline{x}, z)|^{2}\right] \cdot\left\{\operatorname{Re}\left(U, i \frac{\partial}{\partial z} U\right)-(\operatorname{Re} \beta) \cdot(U, U)\right\}^{-1}$

For any pair of functions $U_{1}, U_{2}$ which vanish quickly as $x \rightarrow \infty$ and are periodic in $\mathrm{z}$, with period :

$$
\mathrm{a}=\left|\bar{a}_{3}\right| \text {, we have defined }\left(\mathrm{U}_{1}, \mathrm{U}_{2}\right)=\int \mathrm{d}^{2} \underline{x} \int_{0}^{\mathrm{a}} \mathrm{dz} \mathrm{U}_{1}(\underline{\mathrm{x}}, \mathrm{z}) * \mathrm{U}_{2}(\underline{\mathrm{x}}, \mathrm{z}) \text {, the }
$$

$\underline{x-i n t e g r a t i o n ~ b e i n g ~ e x t e n d e d ~ o v e r ~ a l l ~ t w o-d i m e n s i o n a l ~ s p a c e . ~ S i n c e ~ I m ~} b$ is rather small [2], the replacement of $U$ in the right-handside of Eq. (4) by the propagation modes $U^{(0)}(x, z)$ which correspona to solutions of Eq. (1) with $\operatorname{Im} b=0, \operatorname{Im} v=0$ seems justified: this yields an approximate expres sion for Im $B$ to first order in Im b. As a simple application, we assume a fibre of circular cross section (radius $R$ ) and we recall that well inside an allowed band for the neutron along the fibre, the propagation modes reduce, as a first approximation, to those for the corresponding homogeneous fibre $[1]$, so that $U^{(0)}(\underline{x}, z) \approx \exp i\left(s z /\left|\bar{a}_{3}\right|\right)$, exp $i M \phi . g(r), s$ and $M$ being integers and $g(r)$ being a suitable Bessel (Hankel) function for $r<R(r>R$ ) (see [1], subsections 3.2 and 5.3). Then, it is easy to see that, uncier those assumptions and approximations, the values of $\operatorname{Im} \beta$ for the periodic semiinfinite fibre and for the corresponding hamogeneous one (given in [1], section 4) coincide. Eq. (4) also provides a basis for studying other cases and for improvements.

- As discussed in [4] (sections 2.2 and 2.4), the negative value of the overall amplitude $b$ for the scattering of neutrons by nuclei at thermal energies, $E_{t h}$ is to be associated to the presence of scme resonance levels at $E_{\text {res }}\left(>E_{t h}\right)$ so that the attractive (negative) resonant amplitude predominates over the repulsive (positive) purely potential scattering amplitude: for instance, scattering resonances o.ccur for $\mathrm{Mn}$ at $\mathrm{E}_{\text {res }} \simeq 0.3$ and 2.4 Kev,... A A look at the standard Breit-Wigner formula would suggest that such a predominance and, hence, the negative character of the overall nuclear amplitude $b$ and the possible existence of confinea propagation of neutrons in fibres made up by $T i, M n, .$. should also be valid for neutron energies $\mathrm{E}$ in same range $\mathrm{E}_{1}<\mathrm{E}_{2}<\mathrm{E}_{2}$, where $\mathrm{E}_{1}<\mathrm{E}_{t h}<\mathrm{E}_{2}<\mathrm{E}_{\text {res }}$. This fact may be interesting for those possible applications (like neutron radiotherapy) where is no need that the neutrons propagating along the fibre be monochro matic. 
Notice that the absorption cross section of neutrons by $\mathrm{Ti}$ and other intermediate nuclei, which decreases proportionally as the wavelength does (see pages 122 and 139-140 in [4]), may become smaller for neutron energies somewhat above the thermal range. However, the neutron energy should be kept smaller than about $1 \mathrm{eV}$, in order not to have a large reduction in the thermal neutron flux coming from a reactor, and not to produce damage to the crystal lattice of the fibre.

Acknowledgments. - We are grateful to Prof. J. Bernabéu, Prof. Gomez Sal, Prof. A. Hernando and Dr. S. Noguera for friendly cooperation and interest. We are also grateful to the Organization Committee of the Workshop "Reactor Based Fundamental Physics", Institut Laue-Langevin, Grenoble, for their kind invitation to participate in it.

We acknowledge the partial financial support received from Comision Asesora de Investigación Cientifica y técnica, Spain.

\section{REFERENCES}

[1] Alvarez-Estrada, R.F. and Calvo, M.L., J. Phys. D: Appl. Phys., to appear

[2] Sears V.F. Phys. Reports 82 (1982) 1

[3] Marshall W., Lovesey S.W., Theory of Thermal Neutron Scattering (Clarendon Press), 1971

[4] Bacon, G.E., "Neutron Diffraction" (Clarendon Press), 2nd Edition, 1962

[5] Takei W.J., Shirane G., Frazer B.C., Phys. Rev. 119, (1960), 122

[6] Kittel C., "Introduction to Solia State Physics", 3nd Edition, page 461 (John Wiley) 1963

[7] Wilkinson, M.K., Gringrich N.S., Shull C.G., J. Phys. Chem. Solids 2 (1957) 289

[8] De Alfaro V., Regge T., "Potential Scattering" (North-Holland) page 75, 1975

[9] Berry M.V., Ozario de Almeida A.M., J. Phys. A: Math. Gen. 6 (1973), 1451

[10] Jacrot B., "Utilization of Neutron Guide Tubes for Neutron Ine lastic Scattering", Proc. Symp. Instrumentation for Neutron Inelastic Scattering REsearch, page 225, (IAFA), 1970. 